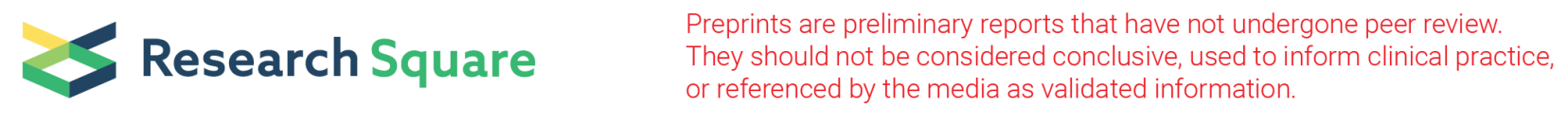

\title{
Paradox of Nonpathogenicity and Cytopathic Effects $\forall$ Characterization of Regulator Tas in Foamy Virus Evolution and De-Ubiquitylation of Tas in Virus Duplication
}

Wei Jie

Shaanxi Normal University

Zhang Rui Fen

Shaanxi Normal University

Song Jing

Shaanxi Normal University

Wu Yan

Shaanxi Normal University

Ma Yong Ping

Shaanxi Normal University

Li Zhi

Shaanxi Normal University

Liu Wan Hong

Wuhan University

He Xiao Hua

Wuhan University

Sun Yan ( $\nabla$ doudousun@sina.com )

Shaanxi Normal University https://orcid.org/0000-0002-4089-4518

\section{Research Article}

Keywords: Foamy virus, Tas, Co-evolution, Ubiquitinion, Immune system

Posted Date: June 15th, 2021

DOI: https://doi.org/10.21203/rs.3.rs-561811/v1

License: (9) (7) This work is licensed under a Creative Commons Attribution 4.0 International License. Read Full License 


\section{Abstract}

Background: Foamy virus, which belong to the Spumaretrovirinae subfamily of Retroviridae, bridge the gap between Orthoretrovirinae and Hepadnaviridae, and display a long co-evolution with their hosts. Like other retroviruses, FVs encode a transactivator, Tas, which governs the levels of viral transcripts initiated through binding to the conserved promoters in $5^{\prime}$ long terminal repeat (LTR) and a unique internal promoter (IP). Unlike the other retrovirus, HIV, foamy viruses induced significant cytopathic effects in vitro, but has no significant disease association at the infection level in vivo. The characterization of regulator Tas in the Paradox of nonpathogenicity and cytopathic effects was still unknown.

Results: Foamy virus separated from different hosts could form three groups which paralleled with the worldwide distribution of hosts due to geographical isolation. Although the physicochemical properties of different Tas were mainly in line with each other, the conserved motifs analyses still suggested divergences in protein functions. The proteins identified to interact with Tas of PFV, SFVora and SFVagm displayed that the three kinds of foamy viruses regulated different signal pathways and impacted virus-host immune interaction. Interestingly, predictions of interactional factors based on protein sequence showed USP7, a kind of deubiquitinating enzyme, could binding directly to Tas which led to its ubiquitin-dependent proteasome degradation. And this results also implyed that fomay virus could hijacked cytokine USP7 to stabilize the transcriptional activator Tas by de-ubiquitylation and thereby regulate the viral life cycle.

Conclusion: Our experiments help to understand the survival strategy of foamy viruses in their hosts.

\section{Background}

Foamy viruses (FVs), named for the cytopathic effect of vacuolation and multinucleation on a variety of cells infected in vitro, were the only genus in the subfamily Spumaretrovirinae of the family Retroviridae and kept co-evolution with hosts for more than 100 million years[1]. FVs occasionally leave the relics of past infections in host genomes which act as "fossils". Recent surveillance on foamy endogenous retroviruses (FERVs) isolated from snakes (Hydrophis hardwickii), birds (Ciconia boyciana)[2], reptiles (Sphenodon punctatus)[3], amphibians (Xenopus tropicalis) and fish (Anoplopoma fimbria) [4]displayed that FVs originated from cross-class transmissions ultimately based on an ancient reptilian animal[5]. Studies on foamy viruses revealed that FVs are highly prevalent among natural mammal hosts, capable of infecting from primates such as marmoset, African green monkey and macaque to equine, feline, bovine and bats[6].

Some typical retroviruses such as human immunodeficiency virus (HIV) and human T cell leukemia virus (HTLV) were kept severe pathogenicity and all verified to be cross-transmission from the counterparts of simian immunodeficiency virus (SIV), simian T cell leukemia virus (STLV)[7]. PFV (prototype foamy virus) was also cross-transmission to human through a zoonotic means in which humans occupationally and naturally exposed to nonhuman primates (NHPs) or lived commensally with NHPs[8]. However, FVs appeared no significant pathogenicity and persisted for long periods with the presence of neutralizing antibodies in the host[9]. In addition, PFV established an asymptomatic infection with specific morphology and provoke characteristic of highly cytopathic in cell culture[10,11]. It was reported that the interaction of host immune-associated cytokines activated by virus invasion led to the virus selfrestriction and shaped the long-term co-evolution of virus-host[12]. As the most important transcriptional regulatory protein of FVs, Tas played a very important role in governing the transcriptions of the structural proteins, initiating virus replication and regulating the interaction between foamy virus and the host cells[13]. But excepted for evolutionary analysis of FVs based on the sequences of structural proteins Gag, Pol, Env or 5'LTR[14], there were few studies on the construction of evolutionary tree based on the function-conserved regulatory protein Tas.

As the transactivator of foamy virus, Tas was reported to be acetylated by HATs p300 and PCAF resulting enhanced ability of DNA binding to promoters and was an effective means to regulate virus transcription $[15,16]$. However, the various post-translational and functional modifications of Tas mediated by the host cell factors and vital for virus-host immunoreactions were still less known. Ubiquitin is one of the most important post-translation modifications (PTMs) which mediated the proteasome degradation pathways in regulating dynamic equilibrium and the stability of substrates[17]. And the degradation efficiency depended on the chain length and the linkage type of ubiquitin, or the susceptibility to deubiquitinase activities[18]. A broad variety of deubiquitinatingenzymes (DUBs) also played the great important role in the ubiquitin proteasome system (USP) which stabilized the substrates by removing the ubiquitin chains to prevent the proteins from proteasomal degradation[19]. HIV-1 Tat was reported to be stabilized by USP7, a kind of typical deubiquitinatingenzymes, which led to the enhancement of gene expressions and viral productions[20]. While for FVs, whether the Tas suffered ubiquitindependent proteasome degradation mediated by deubiquitinatingenzymes USP7 from the host cells was still unknown.

Thus, the aim of our study was to evaluate the natural evolutionary history including genetic diversity of foamy virus based on FVs transactivator Tas sequences and the stability enhancement of Tas mediated by USP7 inhibiting the ubiquitin-dependent proteasome degradation. All these might shed a light to the interactions on the immune reactions during the infections of foamy virus with the host which caused life-long infections in natural hosts without any apparent pathogenicity while significant cytopathic effects in cell culture.

\section{Results}

Phylogenetic analysis and the worldwide distribution of FVs based on Tas revealed co-evolution of foamy viruses and their hosts

Searching for the UniProt databases, we found the complete protein sequences of $12 \mathrm{FVs}$ from 9 primate and 3 non-primate hosts. At the same time, the literatures were consulted to trace the isolation history of different foamy virus from multiple hosts (Table 1). In order to shed light on the evolutionary relationship among these viruses, the phylogenetic analyses were performed based on the different Tas sequences and the cladogram of relative hosts was derived from Timetree databases. According to the comparation of molecular evolution tree (Fig. 1), the 12 sequences formed 2 different monophyletic clusters: all the primate isolates clustered together, while the three non-primate isolates formed the second cluster. Furthermore, as Simian foamy viruses 
(SFVs) are prevalent in all species of non-human primates, we focused on the co-evolution of FVs and their natural non-human primate hosts. The results indicated that SFVagm and SFVmac gathered into a cluster paralleled with Old World monkeys including African green monkey (Cercopithecus aethiops), Macaque (Macaca mulatta). The lineage of SFVora was paralleled with Orangutan (Pongo pygmaeus), another member of Old World monkeys. And Orangutan was placed as a bridge to the apes, which include Chimpanzee (Pan troglodytes verus), Gorilla (Gorilla gorilla sp.) and were in accord with SFVcpz and SFVgor. SFVspm, SFVsqu and SFVmar formed another cluster absolutely consisted with New World monkeys including Spider monkey (Ateles sp.), Squirrel monkey (Saimiri sciureus), Marmoset (Callithrix jacchus). The matching of foamy viruses and their hosts revealed a long-lasting co-speciation history between FVs and ancient primate animals clearly. However, the antinomy appeared that the phylogeny of EFV, FFV and BFV did not mirror the relationship of their relative hosts equine (E), feline (F), and bovine (B), and placed the FFV lineage basal to the EFV and BFV cluster. This marked that the relationships among these hosts are not always consistent among different evolutionary level.

Retroviruses are actively crossing into humans exposed to non-human primates from chimpanzees as well as other non-human primates which led to potential risk of disease, such as simian immunodeficiency virus and simian T lymphotropic virus. However, the origin of retroviruses was still not well documented. To take this line of inquiry a step further on the prevalence of FVs around worldwide, we investigated the distribution of the relative hosts which offered the isolation for different viruses in a variety of contexts, in several countries and from multiple non-human primates (Fig. 2). As the map showed that the worldwide distribution of SFVagm, SFVmac and SFVora were always in keeping with the habitat range of Old World monkeys.

SFVspm, SFVsqu and SFVmar were also consisted with New World monkeys geographically which included tropical regions of Central and South America. Apes whose geographical distribution is mainly concentrated in equatorial tropical forests of Africa took the major responsibility for cross-species transmission from Chimpanzees to humans. But for BFV, EFV and FFV which isolated from equine (E), feline (F), and bovine (B), there is no explicit correlations at the distribution among FVs and their non-primate hosts.

\section{Comparison of physicochemical properties of the three different host-derived foamy virus Tas}

The results of multiple sequence alignment performed by Clustal $X$ displayed that Tas of foam viruses isolated from different hosts varied with each other dramatically (Fig. 3). Several conserved sites were only found either on the middle or the C-terminal of the Tas sequences. And these results displayed that functional fragments of different Tas were still conserved but the tremendous diversities on the other areas could be attributed to that foamy viruses isolated from different hosts suffered disparate challenges for virus survival strategy.

To further compare the functions of different Tas, physicochemical properties were analyzed by many online prediction websites. All of the 12 Tas were detected to have several prominent hydrophobic regions according to the hydrophobicity analysis (supplementary materials 1 ). Except for the foamy viruses isolated from non-primate hosts which were predicted to possess one dominating hydrophilic region and $2 \sim 4$ misty hydrophobic regions, the rest Tas of FVs isolated from primate hosts all possessed three prominent hydrophobic regions and two hydrophilic regions. And the average hydrophobicity results indicated that the Tas protein was hydrophilic.

The physicochemical properties such as theoretical pl, estimated half-life, instability index, aliphatic index and grand average of hydropathicity were also predicted by several online website (Table 2). The theoretical PI values of Tas proteins of these different host-derived foamy viruses ranged from 5.02 to 6.09 . Stability coefficient analysis shows that Tas proteins of other host-derived foamy viruses tend to be unstable except for BTas (Instability Index< 40 ). What's more, there was no significant difference in the prediction of half-life, fat index and other characteristics. The prediction and analysis of Tas secondary structure showed thata-helix and random coil are the main structural components, and $\beta$-corner appears in local regions (supplementary materials 2 ). The proportion of random coils in the secondary structure of Tas protein of foamy virus isolated from different host sources was $60.06 \%-71.67 \%$, the proportion of a-helix was $8.13 \%-32.19 \%$, and the proportion of lamella was $4.32 \%-25.84 \%$ (Table 3 ). All these above showed that although there were much more divergences of amino acid primary structures of different Tas, they still were inclined to keep physicochemical properties of great similarities which lay the foundation for the conservation of motifs initiating the replication of FVs.

Furthermore, the analysis of conserved motifs of foamy viruses, which were secondary units always gathered together to form relatively stable motifs and exert special functions, implied the functional similarity of Tas. And Tas were analyzed using MEME v4.9 online analysis software, and a total of 10 predicted motifs were obtained (Fig. 4). As the diagram displayed that the numbers and types of motifs contained in Tas were shared high similarity. It is concluded that the diversity of motif was related to the various pathways in virus-host immune response, which implied that although faced with different host selection pressure, functional domains are still conserved. What's more, the motifs of functional classification also consist with the results from phylogenetic analyses which were divided into branches of simian foamy virus from the primate hosts. All these suggested the exist of co-species except for the cross-species transmission.

\section{Interactor enrichment analysis of the three foamy virus Tas isolated from different host origins}

Although we had already found that various Tas of foamy virus isolated from different host was absolutely diversity, the function of motifs was verified conserved. Therefore, proteomics were performed in the stable cell lines with Tas of PFV $\square$ SFVagm and SFVora overexpression which are different but much closer to that of PFV in motifs analysis to detect the differences of specific protein interactions. Finally, we detected 65,67 and 121 proteins in the overexpressed PFV-tasM, SFVagm-tasM and SFVora-tasM separately (Supplementary Material 3). Through GO analysis, the enriched proteins related to

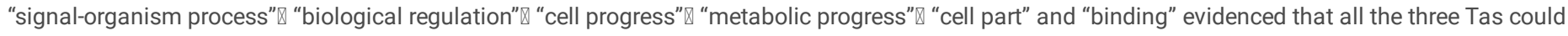
widely affected Biological Process, Cell Component and Molecular Function (Fig. 5a). Furthermore, it was verified that the three kind of Tas regulated different 
cell signaling pathways by KEGG enrichment analysis (Fig. 5b). For Tas of PFV, the most significant enrichment pathway was "Basal transcription factors" while "Central carbon metabolism in cancer" for SFVagm Tas and "DNA replicating" for SFVora Tas. And the protein-protein interactions (PPI) network for PFV and SFVagm Tas were concentrated to histone acetylation, but for SFVora Tas, proteins functioned on "positive regulation of DNA metabolic process" $]$ "telomere maintenance" $\rrbracket$ “Cell Cycle" were concentrated(Fig. 5c). All these implied that the three kind of Tas proteins regulated different cellular effects by inducing different signaling pathways in the virus-host cell immune response.

\section{Interactions with USP7 illustrated the ubiquitin-dependent proteasome degradation of the Tas}

By further comparing the types of cytokines interacted with Tas proteins of foamy viruses derived from different host origin, we had found that all the three kinds of Tas proteins could bind ubiquitin-proteasome degradation pathway related proteins PSMD11 (components of the 26S proteasome) and TRIM21(E3 ubiquitin protein ligase) (Fig. 5d). Therefore, we had conjectured that all the three kinds of Tas proteins were encountered the antagonism of host degradation mediated by ubiquitination which might play an important role in the invasion removement of the foamy viruses. Linear motifs are short components which provide low-affinity interaction interfaces and play central roles in mediating the regulatory functionality of the cell. They are particularly controlling protein turnover during the infection of viruses and always lead to the activation of host cells signaling. Here we employed the Eukaryotic Linear Motif (ELM) resource to discover putative linear motifs in Tas of SFVagm, SFVora and PFV. As the predictions showed that there were many putative binding sites which could

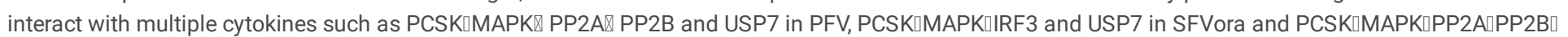
APCC and USP7 in SFVagm (Fig. 6a). At the same time, as the stable expression of reverse transcription regulator Tas was the precondition of FVs replication and important manifestation of virus-host immune reaction, we focused on USP7 for its unique deubiquitylation-functions in the ubiquitin-proteasome degradation pathway. We performed immunoprecipitation test to detected the accuracy of prediction results and verified that all the three kinds of Tas could interact with USP7 as predicted in vivo (Fig. 6b). To investigate whether in the absence of P5091, a specific inhibitor of the deubiquitinase enzymes USP7, the degradation of Tas proteins were occurring through the proteasomal pathway, pcDNA ${ }^{\mathrm{Tm}} 4 / \mathrm{myc}_{\mathrm{m}} \mathrm{His}$ A-SFVagm-Tas, pcDNA ${ }^{\mathrm{Tm}} 4 / \mathrm{myc}^{-H i s} \mathrm{~A}-\mathrm{SFV}$ ora-Tas and $\mathrm{pcDNA}^{\mathrm{Tm}} 4$ /myc-His A-PFV-Tas transfected Hela cells were treated with P0591. The results showed that all Tas underwent the fate of degradation invariably (Fig. 6c, upper). Furthermore, as the increasing of the concentration of P0591, much more Tas proteins were urged into degradation which indicated a dosedependent decrease of Tas proteins. What's more, when MG132 was treated with the Tas proteins overexpression showed a slight increase in Tas level suggested that all the three kind of Tas proteins suffered of proteasome degradations (Fig. 6c, bottom). All these suggested that the three kinds of Tas proteins could all hijack USP7 to stabilize themselves and regulate foamy viruses replication.

\section{Discussion}

To better understand foamy virus "zoonosis" in natural settings and further evaluated the relationship between foamy virus and the hosts immune reactions, the coevolutionary analysis of foamy viruses with the hosts and their interaction with the host cells had been explored deeply based on the transactivator Tas.

By looking back on the history of FVs isolations, we had found that infections of FVs on simians were more prevalent and primary which supported the origin theory of the transmission from non-human primates to other hosts. And the phylogenetic tree based on Tas or the worldwide distribution of foamy viruses were all parallel to the cladogram derived from the host animals which were a prerequisite to the assumption of co-speciation and affirmed the co-evolution of FVs and their hosts. But the replacement rate of FVs infectious among different hosts was not even which ranged from 0.094 to 0.993 , especially SFVsqu whose replacement rate was up to $99.3 \%$. This implied that although the rate of foamy viruses spread and mutation were generally related to the evolutionary status of the host, it still closely related to the reproductive cycle and population size of the host species. The longer the generation, the larger the population, and the slower the mutation. In another words, the more adapted foamy virus was to a particular environment, the less likely it would be able to adapt to a new environment, and the more likely it was to reach an evolutionary equilibrium. Although the primary structure of different Tas analyzed by multiple sequence alignment was less conserved, the physicochemical properties which represented the unique and fundamental characteristic of proteins such as hydrophobicity analysis, theoretical pl, estimated half-life, instability index, aliphatic index and grand average of hydropathicity were all broadly consistent with each other. Furthermore, similarity analysis of conserved motifs of different Tas sequences displayed the consistency with the results from phylogenetic and the worldwide distribution analyses. All these suggested that the diversity of motifs was related to the various pathways in virus-host immune response due to the different infection environments offered by the host animals. However, the transactivation function of Tas initiated FVs replications were conserved when encountering different evolutionary selection pressures in the process of leaping transmission species. Therefore, we could conclude that foamy viruses were divergent due to cross-transmission accidently through saliva-dependent means and afterwhile were also separated for the geographical distribution. All these led to the species-specific co-speciation for a long time which was also asserted that foamy viruses had co-speciated with Old World primates for at least 30 million years[1]. It is widely accepted that PFV is the fact of cross-species transmission from chimpanzee to human rather than co-speciation[21]. And although latent proviruses can be detected in most tissues, foamy virus replication is still limited to the oral mucosa under natural infection[22]. It implied that not all interspecies transmissions led to successful onwards transmission in new hosts, and co-speciation remains still holds as major long-term evolutionary event. Due to the virus lineage was continually transmitted between different host lineages, horizontal transmission like cross-transmission impeded tight coevolution between a single host and the virus lineage[23]. But, vertical transmission such as co-speciation provided a unique opportunity for strong coevolution, because host and the virus lineages were paired over multiple generation and antagonism was perfectly evolved[24]. As a typical retrovirus, foamy viruses integrated their genetic information into host cell chromosomes. The unique self-replication mechanism took advantage of complex repair systems of host cell to help foamy viruses avoided the introduction of many RT induced mutations over short time[25]. The co-evolution balance between foamy viruses and their host revealed the reason why foamy viruses did not cause diseases at the organism level. However, the phylogenetic tree of nonprimate EFV, FFV and BFV were neither mirrored the lineage of their counterpart hosts equine (E), feline (F), and bovine (B), nor the distribution around the worldwide. This could be explained by preferential host switching which suggested that viruses are more likely to jump between related host species[21]. As 
non-primate hosts always kept by humans for pets or the agricultural economy, the possibility of such inter-species transmission caused by foamy viruses of live-stock or companion animals which subjected to human intervention and escaped from natural selection may be another reason.

In order to deeply investigate the virus-host immune interaction, we focused on cytokines interacted with Tas proteins of SFVagm, SFVora which were different but much closer to that of PFV in motifs analysis. Finally, we detected that the three different kinds of Tas proteins could all combine with the ubiquitinproteasome degradation system relative protein PSMD11 and TRIM21, we conjectured that proteasome degradation mediated by ubiquitin played a basial function during infection of foamy viruses on the host cells. And Eukaryotic Linear Motif (ELM) was used to predict low-affinity interactions between Tas and other cytokines which may be of great importance for modification of Tas and virus-host immune reactions[26]. As the Fig. 6 showed that USP7, a kind of deubiquitinating enzymes (DUBs), was predicted to interact with Tas from SFVagm, SFVora and PFV at the putative binding sites. As one of the most important post-translation modifications (PTMs) which was responsible for the regulation of the stability and activity of virous modified proteins, ubiquitin was attached to the target substrates and activated by an E1 enzyme during an ATP-dependent reaction. Then the complex was conjugation by an E2 class enzyme which followed by E3 ubiquitin ligase to ubiquitinate target proteins directly or indirectly[17]. Ubiquitin-dependent modification led to the proteasome degradation and the dynamic balance of proteins not only in cell cycles but also in virus replication[27]. Specifical chemical inhibitors (P0591) of USP7 were treated to verify the impact on different Tas. The results proved that USP7 enhanced the stabilization of Tas and prevent it from ubiquin-dependent proteasome degradation. However, unlike the tat of HIV-1, which was also stabilized by USP7 in the process of virus infection, the level of USP7 was not influenced by Tas in turn[20]. This may be a kind of effective virus survival strategy that FVs hijacked the host cell cytokines to escape from the immune antagonist and try the best to intensify self-proliferation and produce the largest number of progeny viruses.

\section{Conclusion}

Combined the results from the phylogenetic tree based on Tas protein sequences and the worldwide distribution of different foamy viruses, it is confirmed that foamy viruses and their hosts had experienced a long time of evolution. The physicochemical properties of different Tas are generally consistent with each other, while the conserved motifs in Tas and the cellular signal pathways stimulated by Tas proteins are somewhat diverse. Further study revealed that Tas could interact with USP7 to protect itself from the ubiquitin-dependent degradation pathway which may regulate the immune-interaction during foamy virus infection. Our experiments help to understand the survival strategy of foamy viruses in their hosts.

\section{Methods}

Bioinformatics analysis

The sequences analyzed in this article were all obtained by searching the UniProt database (https://www.uniprot.org/). MAFFT 7.0 was used to perform highprecision multi-sequence alignment of Tas protein sequences isolated from different host sources. Clustal $X$ software was used to combine the amino acid sequences, and a phylogenetic tree was constructed using the Neighbor-linkage method (NJ) by Mega V4.0. The host evolutionary tree is derived from the Timetree database (http://timetree.org/). The hydrophobicity analysis of Tas was made with the ExPASy Proteomics Server using thescale Hphob. /Kyte \& Doolittle (http://web.expasy.org/cgi-bin/prots cale/protscale.pl). The prediction of the secondary structure of Tas was made using Secondary Structure Prediction server - PHD (http://npsa-pbil.ibcp.fr/cgi-bin/npsa_automat.pl?page=/NPSA/npsa_phd.html). The predictions of physicochemical properties of Tas were made with the online program ProtParam (https://web.expasy.org/protparam/). All Tas sequences and functional domain predictions were performed by MEME v4.9(http://meme.nbcr.net/Meme/cgi-bin/meme.cgi). EML (http://elm.eu.org) was used to discover functional linear motifs of Tas that potentially interacted Eukaryotic cytokines.

\section{Cell culture, Plasmids and Transfection:}

HeLa cells were cultivated in Dulbecco's modified Eagle's medium (12100, Solarbio, China) supplemented with $1 \%$ penicillin and streptomycin and $10 \%$ fetal bovine serum (CP17-1616, Capricorn, Germany) in $5 \% \mathrm{CO}_{2}$ at $37^{\circ} \mathrm{C}$. The gene sequences of SFVagm, SFVora which derived from NCBI were synthesized by Genewiz. Lenti-X Lentiviral Expression Systems were used to perform the doxycycline-inducible $3 \times F$ lag-Tas of SFVagm, SFVora and PFV in Hela cell absolutely followed User Manual. The recombinant plasmid pcDNA ${ }^{\mathrm{T}} 4 / \mathrm{myc}-\mathrm{His}$ A was constructed to overexpress Tas of SFVagm, SFVora and PFV and were transfected into Hela cells with lipofectamine 2000 following the manufacturer's protocol. USP7 inhibitor P5091 and proteasomal inhibitor MG132 were purchased from Sigma Aldrich, USA and dissolved in dimethyl sulphoxide (DMSO) was added into cell mediums at different concentrations.

\section{Western blotting:}

Cell lysates was extracted from the cell lines with a RIPA lysis buffer (150 mM NaCl, 0.5\% sodium deoxycholate, 0.1\% SDS, 1mM EDTA, 50mM Tris-HCl, pH 7.4, $1 \mathrm{mM}$ PMSF) and was quantified by using the BCA kit (Thermo Fish). And then Samples were resolved by SDS/PAGE (polyacrylamide gel electrophoresis) and transferred onto the polyvinylidene difluoride (PVDF) membrane (PALL, USA). The membranes were blocked with $5 \%$ nonfat milk, then incubated with primary antibodies at $4^{\circ} \mathrm{C}$ overnight. After incubated with either goat anti-rabbit or goat anti-mouse secondary antibody conjugated with horseradish peroxidase (HRP) for $60 \mathrm{~min}$, membranes were treated with enhanced chemiluminescence reagents (Tanon, China). Protein signals were detected by image analysis system (Tanon Corp, Tanon 5200, China). The primary antibodies were used as follows: Myc (67447-1-Ig, Proteintech), GAPDH (60004-1-Ig, Proteintech), Flag (660083-Ig, Proteintech), USP7 (\#4833, CST). 


\section{Co-immunoprecipitation:}

For protein-protein interaction analysis, Hela- SFVagm-3×Flag-Tas, Hela- SFVora-3×Flag-Tas, Hela- PFV-3×Flag-Tas cells were seeded into 10-cm dish for 24h and then added Dox into the cell medium at $1 \mathrm{ug} / \mathrm{ml}$ for the overexpression of Tas for $48 \mathrm{~h}$. Cells were harvested and lysed in immunoprecipitation buffer (50mM Tris, $150 \mathrm{Mm} \mathrm{Nacl}, 1 \mathrm{Mm}$ EDTA, $1 \%$ Triton-100, 25xprotease inhibitor cocktails) for $30 \mathrm{~min}$, and then centrifuged at $13000 \mathrm{~g}$ for $10 \mathrm{~min}$ at $4^{\circ} \mathrm{C}$. Thereafter, supernatants were incubated with indicated antibodies (1ug) overnight at $4^{\circ} \mathrm{C}$, and then rotated with Protein A-agarose (P27531, Yeasen, China) for $4 \mathrm{~h}$ at $4{ }^{\circ} \mathrm{C}$. After being washed with lysis butter for 6 times, the immunoprecipitation materials were boiled in 40ul 1×SDS loading butter and subjected to western blotting using indicated antibodies.

\section{Declarations}

Ethics approval and consent to participate Not applicable.

Consent for publication Not applicable.

Availability of data and materials Data sharing is not applicable to this article as no datasets were generated or analysed during the current study.

Competing interests The authors declare that they have no competing interests

Funding This work was supported by the grants from the National Natural Science Foundation of China (No. 31670163), the Fundamental Research Funds for the Central Universities (GK201902010, GK202007019), and the Innovative Experiment Projects of Educational Ministry of China for Undergraduate (cx2019098).

Authors' contributions SY* and LZ designed the experiments. WJ conducted the experiments, analyzed the data and prepared the manuscript. ZRF and MYP performed Co-IP experiment, WY and SJ constructed the phylogenetic tree. LWH and HXH gave suggestions on the study.

*These authors contributed equally to this work as co-corresponding authors

Acknowledgements The author would like to thank Dr. Guandelong for his advices on the co-evolution of foamy viruses and their hosts.

\section{References}

1. Hayward A. Origin of the retroviruses: when, where, and how? Curr Opin Virol. 2017;25:23-7.

2. Aiewsakun P. Avian and serpentine endogenous foamy viruses, and new insights into the macroevolutionary history of foamy viruses. Virus Evol. 2020;6(1):vez057.

3. Wei X, Chen Y, Duan G, Holmes EC, Cui J. A reptilian endogenous foamy virus sheds light on the early evolution of retroviruses. Virus Evol. 2019;5(1):vez001.

4. Aiewsakun P, Katzourakis A. Marine origin of retroviruses in the early Palaeozoic Era. Nat Commun. 2017;8:13954.

5. Aiewsakun P, Richard L, Gessain A, Mouinga-Ondeme A, Vicente Afonso P, Katzourakis A. Modular nature of simian foamy virus genomes and their evolutionary history. Virus Evol. 2019;5(2):vez032.

6. Meiering CD, Linial ML. Historical perspective of foamy virus epidemiology and infection. Clin Microbiol Rev. 2001;14(1):165-76.

7. Charleston MA, Robertson DL. Preferential host switching by primate lentiviruses can account for phylogenetic similarity with the primate phylogeny. Syst Biol. 2002;51(3):528-35.

8. Aris Katzourakis PA, Hongwei Jia ND, Wolfe M, LeBreton AD, Yoder, William M, Switzer. Discovery of prosimian and afrotherian foamy viruses and potential cross species transmissions amidst stable and ancient mammalian co-evolution. 2014.

9. Robert Rustigian PJ, Reihart H. Infection of monkey kidney tissue cultures with virus-like agents. 1955.

10. Judy. Mikovits pmHar. In vitro infection of primary and retrovirus-infected human leukocytes by human foamy virus. 1996.

11. Lo YT, Tian T, Nadeau PE, Park J, Mergia A. The foamy virus genome remains unintegrated in the nuclei of G1/S phase-arrested cells, and integrase is critical for preintegration complex transport into the nucleus. J Virol. 2010;84(6):2832-42.

12. Gibson AK, Stoy KS, Gelarden IA, Penley MJ, Lively CM, Morran LT. The evolution of reduced antagonism-A role for host-parasite coevolution. Evolution. 2015;69(11):2820-30.

13. Mel Campbell CE. and Paul A. luciw*. The Simian Foamy Virus Type 1 Transcriptional Transactivator (Tas)_Binds and Activates an Enhancer Element in the gag Gene. Journal of Virology. 1996.

14. Wang M, Zhang H, Liu QM, Sun Y, Li Z, Liu WH, et al. Structure of transmembrane subunits gp47 of the foamy virus envelope glycoproteins. Acta Virol. 2016;60(2):181-9.

15. Bannert H, Muranyi W, Ogryzko VV, Nakatani Y, Flugel RM. Coactivators p300 and PCAF physically and functionally interact with the foamy viral transactivator. BMC Mol Biol. 2004;5:16.

16. Bodem J, Krausslich HG, Rethwilm A. Acetylation of the foamy virus transactivator Tas by PCAF augments promoter-binding affinity and virus transcription. J Gen Virol. 2007;88(Pt 1):259-63.

17. Akutsu M, Dikic I, Bremm A. Ubiquitin chain diversity at a glance. J Cell Sci. 2016;129(5):875-80.

Page 6/13 
18. Clague MJ, Urbe S. Ubiquitin: same molecule, different degradation pathways. Cell. 2010;143(5):682-5.

19. McClurg ULRC. Deubiquitinating enzymes as oncotargets. Oncotarget. 2015.

20. Ali A, Raja R, Farooqui SR, Ahmad S, Banerjea AC. USP7 deubiquitinase controls HIV-1 production by stabilizing Tat protein. Biochem J. 2017;474(10):1653-68.

21. Schulze A, Lemey P, Schubert J, McClure MO, Rethwilm A, Bodem J. Complete nucleotide sequence and evolutionary analysis of a gorilla foamy virus. J Gen Virol. 2011;92(Pt 3):582-6.

22. Budzik KM, Nace RA, Ikeda Y, Russell SJ. Oncolytic Foamy Virus - generation and properties of a nonpathogenic replicating retroviral vector system that targets chronically proliferating cancer cells. J Virol. 2021.

23. Wade MJ. The co-evolutionary genetics of ecological communities. Nat Rev Genet. 2007;8(3):185-95.

24. Andrew D, Stewart JMLJ, Steven E, Kelley. An empirical study of the evolution of virulence under both horizontal and vertical transmission. 2005. 25. Holmes EC. The phylogeography of human viruses. Mol Ecol. 2004;13(4):745-56.

26. Via A, Gould CM, Gemund C, Gibson TJ, Helmer-Citterich M. A structure filter for the Eukaryotic Linear Motif Resource. BMC Bioinformatics. $2009 ; 10: 351$.

27. Zhadina M, Bieniasz PD. Functional interchangeability of late domains, late domain cofactors and ubiquitin in viral budding. PLoS Pathog. 2010;6(10):e1001153.

\section{Tables}

Table.1 Tas sequences of different FVs isolated from virous hosts.

\begin{tabular}{|c|c|c|c|c|}
\hline Name & Host & UniProt Number & Provenance & Isolation \\
\hline $\mathrm{BFV}$ & Cow (Bos taurus) & J9VDW6 & $\begin{array}{l}\text { Germany } \\
\text { Poland } \\
\text { Japan } \\
\text { China } \\
\text { USA }\end{array}$ & $\begin{array}{l}1978 \\
2005 \\
2018 \\
1997 \\
/\end{array}$ \\
\hline FFV & Domestic cat (Felis catus) & 56862 & $\begin{array}{l}\text { Turkish } \\
\text { UK } \\
\text { UK (Cat sarcoma) } \\
\text { Japan } \\
\text { USA }\end{array}$ & $\begin{array}{l}2019 \\
1997 \\
1969 \\
2000 \\
1975\end{array}$ \\
\hline EFV & $\begin{array}{l}\text { Horse (broodmare) } \\
\text { (Equus ferus caballus) }\end{array}$ & Q9J4C5 & $\begin{array}{l}\text { France } \\
\text { Japan }\end{array}$ & $\begin{array}{l}2000 \\
2019\end{array}$ \\
\hline SFVspm & Spider monkey (Ateles sp.) & A8HC80 & $\begin{array}{l}\text { USA } \\
\text { Germany }\end{array}$ & $\begin{array}{l}1973 \\
2007\end{array}$ \\
\hline SFVsqu & $\begin{array}{l}\text { Squirrel monkey } \\
\text { (Saimiri sciureus) }\end{array}$ & D5JWV8 & Brazil & 2013 \\
\hline SFVmar & $\begin{array}{c}\text { Marmoset } \\
\text { (Callithrix jacchus) }\end{array}$ & D5JWV3 & USA & 1981 \\
\hline SFVagm & $\begin{array}{c}\text { African green monkey } \\
\text { (Cercopithecus aethiops) }\end{array}$ & P27402 & Africa & 1979 \\
\hline SFVmac & $\begin{array}{c}\text { Macaque } \\
\text { (Macaca mulatta, M. cyclopis) }\end{array}$ & P29169 & $\begin{array}{c}\text { Cambodia } \\
\text { Philippines } \\
\text { USA (domestic breeding colony in Morgan Island, SC) } \\
\text { Taiwan } \\
\text { Japan } \\
\text { Cameroon }\end{array}$ & $\begin{array}{l}2013 \\
2016 \\
2017 \\
2018 \\
2020 \\
2010\end{array}$ \\
\hline SFVora & $\begin{array}{c}\text { Orangutan } \\
\text { (Pongo pygmaeus) }\end{array}$ & Q7SIS4 & $\begin{array}{c}\text { UK } \\
\text { Indonesia: Borneo/Sumatra }\end{array}$ & $\begin{array}{l}1994 \\
2003\end{array}$ \\
\hline SFVgor & $\begin{array}{c}\text { Gorilla } \\
\text { (Gorilla gorilla sp.) }\end{array}$ & E2GD60 & $\begin{array}{l}\text { Cameroon } \\
\text { Gabon }\end{array}$ & 2004 \\
\hline SFVcpz & $\begin{array}{c}\text { Chimpanzee } \\
\text { (Pan troglodytes verus) }\end{array}$ & Q87042 & Cameroon & 1996 \\
\hline PFV & $\begin{array}{c}\text { Human } \\
\text { (Homo sapiens) }\end{array}$ & $\overline{P 14353}$ & Kenyan & 1971 \\
\hline
\end{tabular}

Table.2 physicochemical properties of FVs Tas isolated from virous hosts 


\begin{tabular}{|c|c|c|c|c|c|c|c|c|c|}
\hline Virus & PFV & SFVcpz & SFVgor & SFVora & SFVmac & SFVagm & SFVmar & SFVsqu & SFVspm \\
\hline $\begin{array}{l}\text { Number of } \\
\text { amino acids } \\
\text { (aa) }\end{array}$ & 300 & 300 & 299 & 278 & 300 & 298 & 305 & 334 & 324 \\
\hline \multicolumn{10}{|l|}{$\begin{array}{l}\text { Molecular } \\
\text { weight (Da) }\end{array}$} \\
\hline \multicolumn{10}{|l|}{ Theoretical pI } \\
\hline & 5.61 & 5.97 & 5.82 & 5.37 & 6.09 & 5.62 & 6.02 & 5.25 & 5.02 \\
\hline \multicolumn{10}{|l|}{$\begin{array}{l}\text { The estimated } \\
\text { half-lifeelhours }\end{array}$} \\
\hline \multicolumn{10}{|l|}{$\begin{array}{l}\text { Instability } \\
\text { index }\end{array}$} \\
\hline \multicolumn{10}{|l|}{$\begin{array}{l}\text { Aliphatic } \\
\text { index }\end{array}$} \\
\hline $\begin{array}{c}\text { Grand } \\
\text { average of } \\
\text { hydropathicity } \\
\text { (GRAVY) }\end{array}$ & -0.643 & -0.637 & -0.574 & -0.655 & -0.743 & -0.758 & -0.681 & -0.569 & -0.801 \\
\hline
\end{tabular}

Table.3 Secondary structure composition of FVs Tas isolated from virous hosts

\begin{tabular}{cccc}
\hline \multirow{2}{*}{ Virus } & \multicolumn{3}{c}{ Secondary structure composition } \\
\cline { 2 - 4 } & Alpha helix (\%) & Extended strand (\%) & Random coil (\%) \\
\hline PFV & 14.33 & 16.67 & 69 \\
SFVcpz & 13.67 & 14.67 & 71.67 \\
SFVgor & 19.73 & 13.04 & 67.22 \\
SFVora & 26.26 & 8.63 & 65.11 \\
SFVmac & 30.19 & 9.74 & 60.06 \\
SFVagm & 25.5 & 10.07 & 64.43 \\
SFVmar & 32.13 & 6.56 & 61.31 \\
SFVsqu & 23.65 & 6.89 & 69.46 \\
SFVspm & 29.01 & 4.32 & 66.67 \\
BFV & 16.47 & 15.66 & 67.87 \\
EFV & 18.07 & 12.27 & 64.66 \\
FFV & 8.13 & 25.84 & 66.03 \\
\hline
\end{tabular}

\section{Figures}




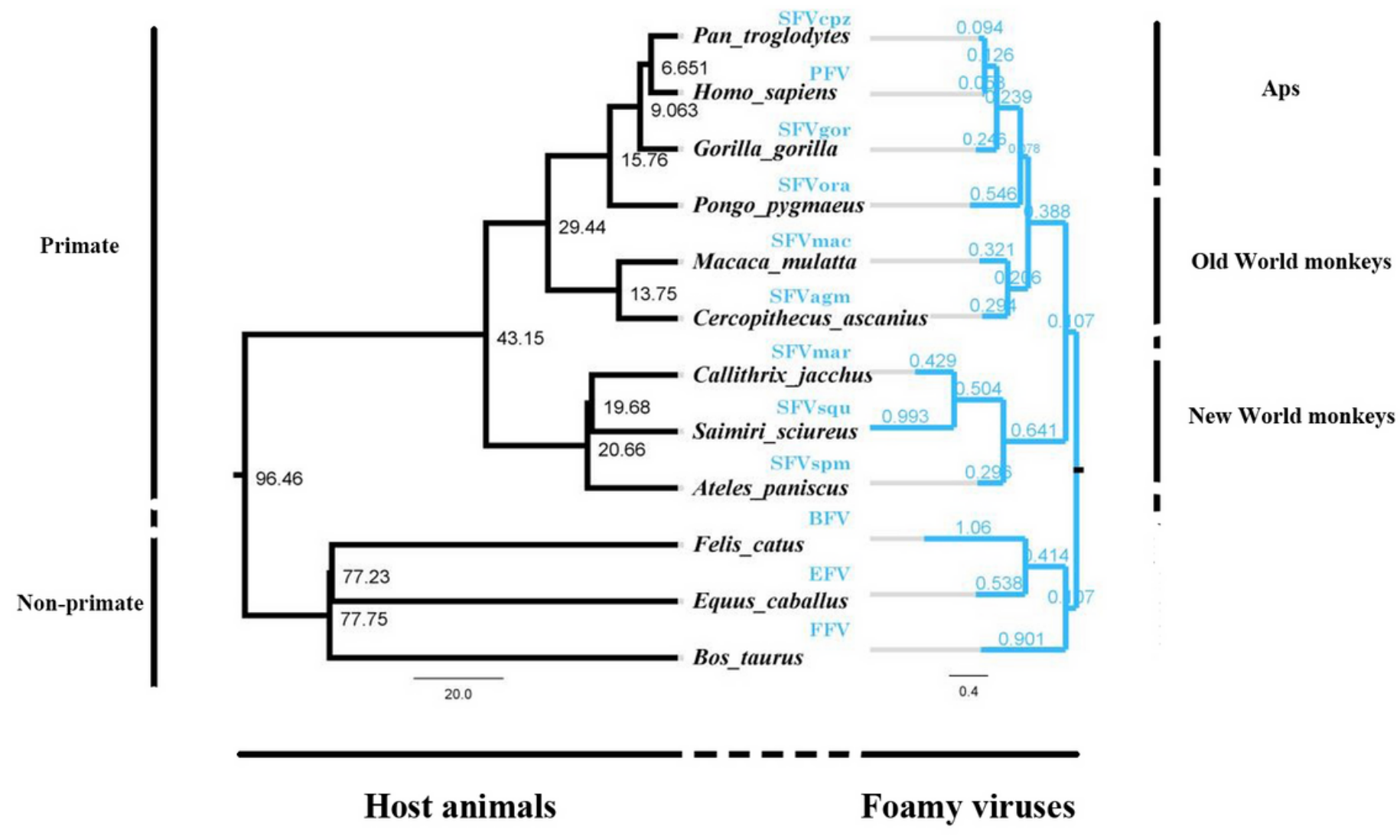

Figure 1

Phylogenetic analysis of FVs based on Tas revealed co-evolution of foamy viruses and their hosts. The schematic diagrams of different Tas sequences from virous FVs isolated from relative hosts were used to perform the phylogenetic trees (the right branches in blue) and Clustal X software was used to combine the amino acid sequences, and a phylogenetic tree was constructed using the Neighbor-linkage method (NJ) by Mega V4.0, and the cladogram of relative hosts were derived from the animal evolutionary database Timetree (http://timetree.org) (the left branches in black). Numeric character up each branch represented different amino acid substitution rate. 
A worldwide distribution of foamy virus' hosts

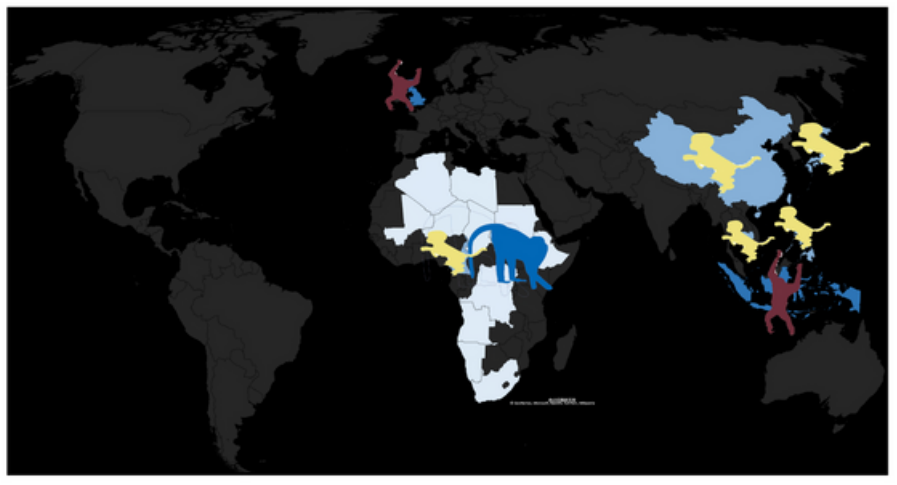

LC Cercopithecus aethiops

A worldwide distribution of foamy virus' hosts

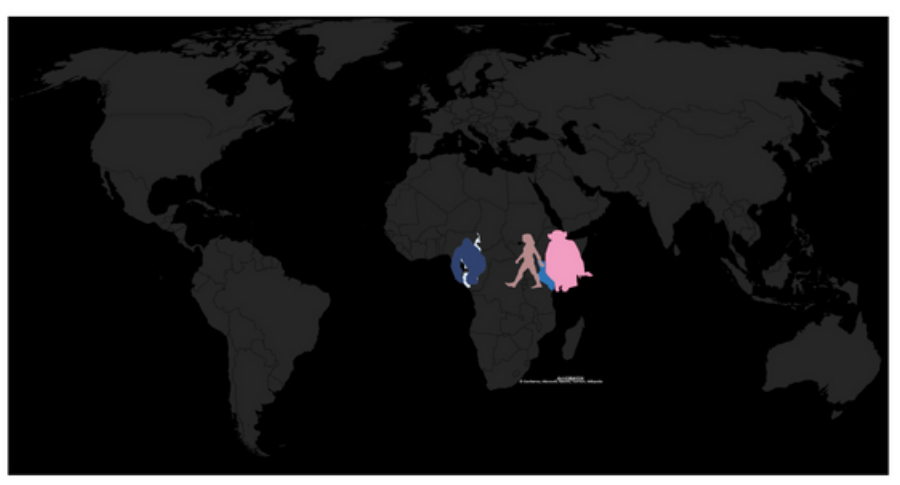

Pan troglodytes verus

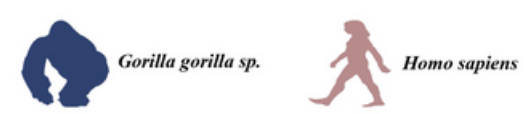

A worldwide distribution of foamy virus' hosts
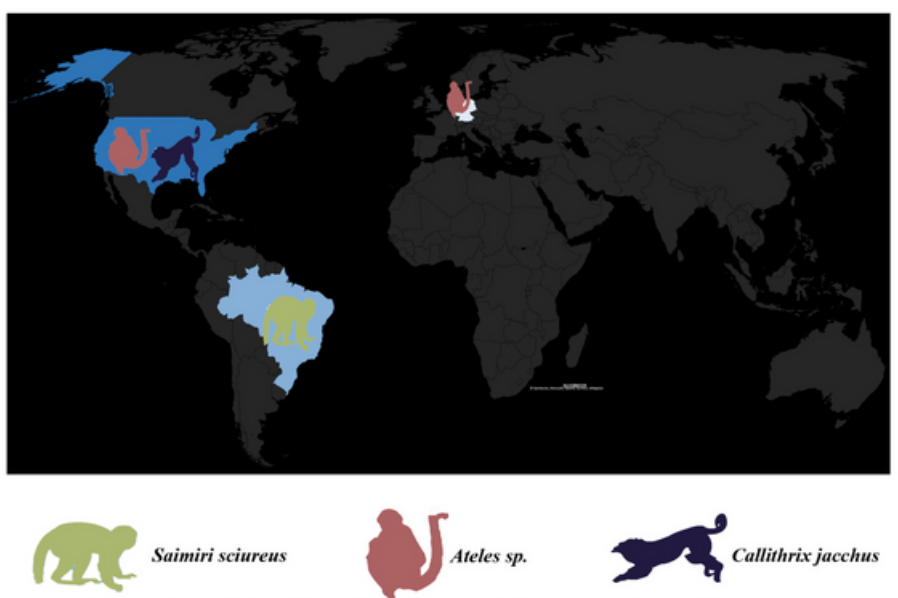

A worldwide distribution of foamy virus' hosts

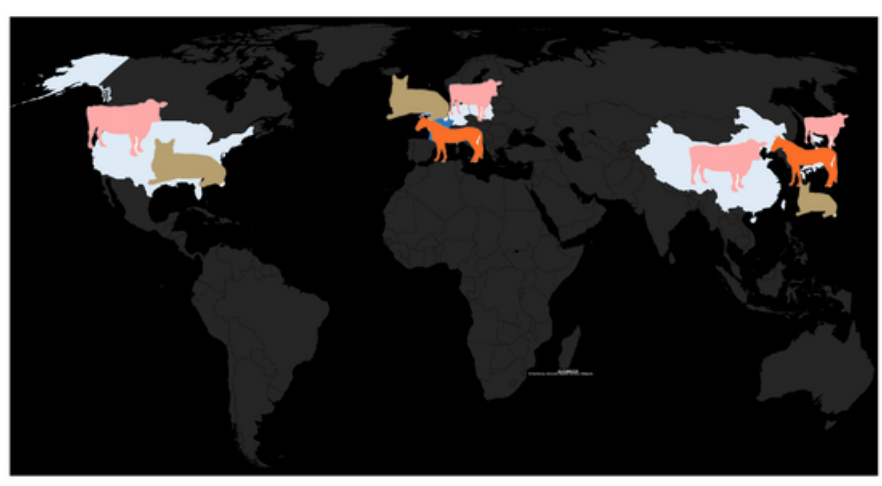

$\sqrt{12}$ Bos taurus

Figure 2

The worldwide distribution of FVs and their relative hosts revealed co-evolution of foamy viruses and their hosts. The complete protein sequences of $12 \mathrm{FVs}$ from 9 primate and 3 non-primate hosts was surveyed in the UniProt (http://uniprot.org). And the isolation histories of different FVs were further tracking, including the distribution of the FVs and their relative hosts. The map was labeled by different cartoon image which represented FVs isolated from the different host animals. And the areas filled with different blue represent the ecological niche of their relative hosts. Note: The designations employed and the presentation of the material on this map do not imply the expression of any opinion whatsoever on the part of Research Square concerning the legal status of any country, territory, city or area or of its authorities, or concerning the delimitation of its frontiers or boundaries. This map has been provided by the authors.

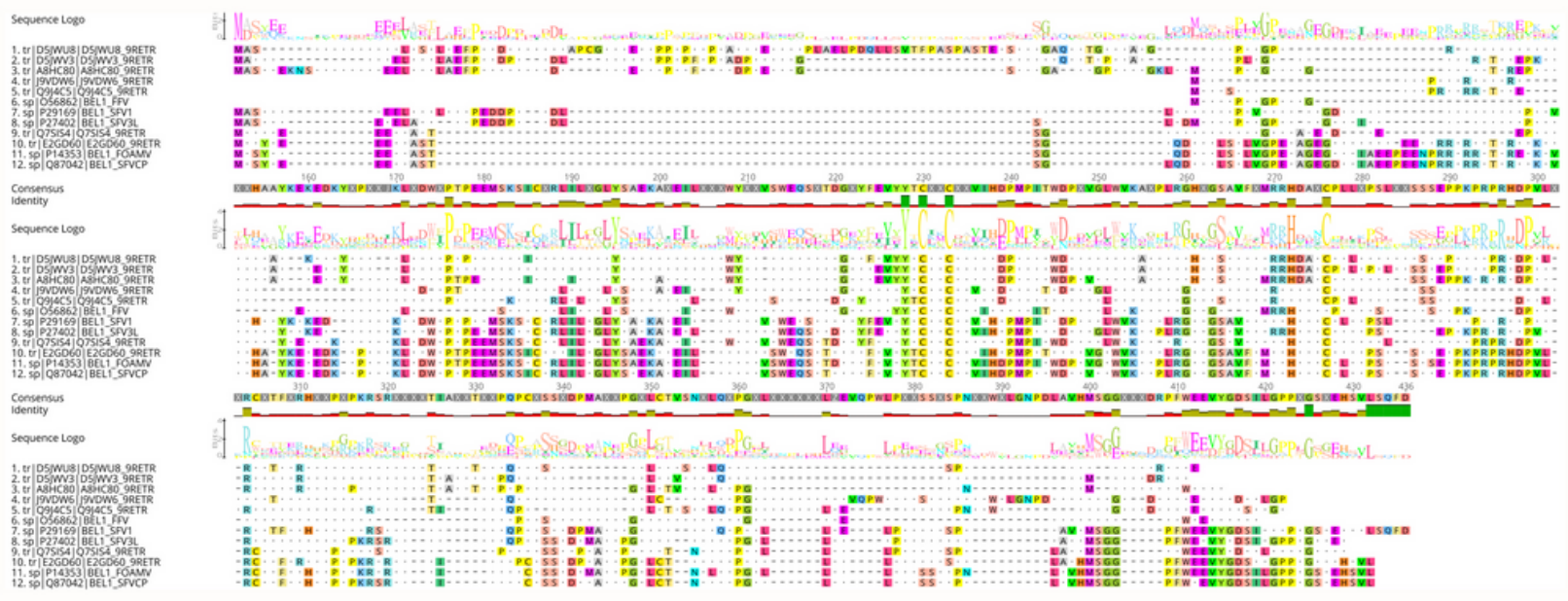

Figure 3 
Multiple sequences analyses of virous FVs Tas isolated from different hosts displayed the low conservation. The sequences analyzed in this article were all obtained by searching the UniProt database (https://www.uniprot.org/). MAFFT 7.0 was used to perform high-precision multi-sequence alignment of Tas protein sequences isolated from different host sources. The neater the different amino acids were, the bigger the logos upon the arrangement were and the more conservative the different Tas were.

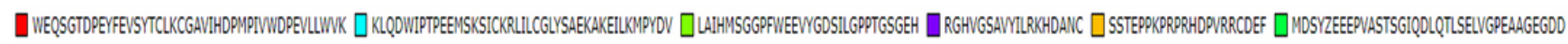

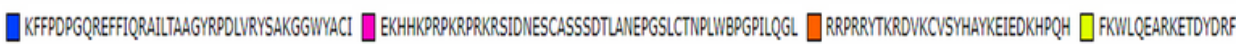

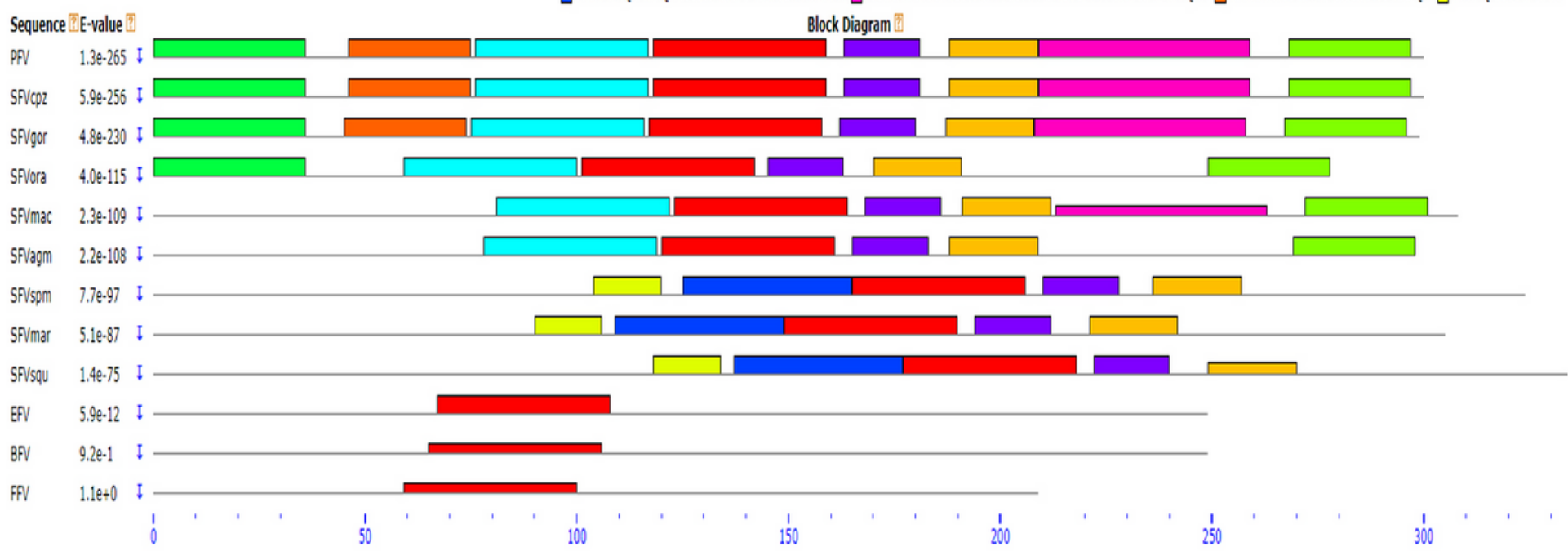

\section{Figure 4}

Predicational motifs analyses of virous FVs Tas isolated from different hosts displayed the functional conservation. All the above 12 Tas sequences and functional domain predictions were performed by MEME v4.9(http://meme.nbcr.net/Meme/cgi-bin/meme.cgi). Virous colors represent different conserve motifs predicted by the website and functional conservation was found to be parallel with phylogenetic trees. 


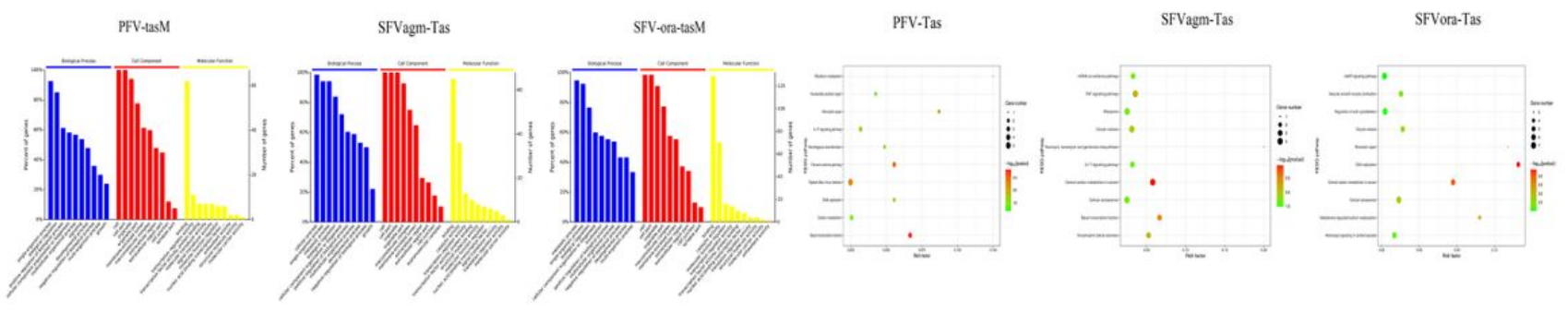

c

PFV-Tas

SFVagm-Tas

SFVora-Tas
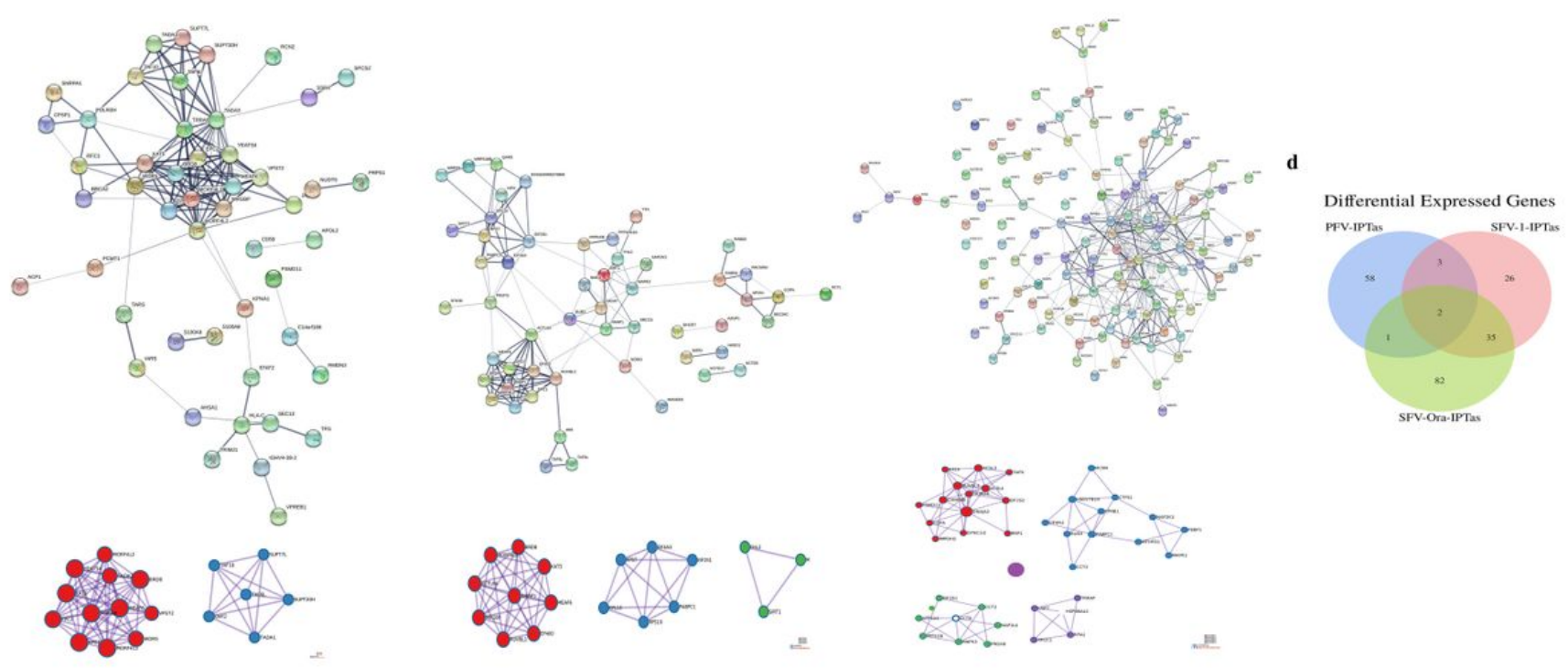

Figure 5

Transcriptome analyses of MOF@FM induced DCs. a, The identified proteins interacted with Tas of PFV, SFVora and SFV agm which associated with biological process, cellular component, and molecular functions based on GO annotation. b, KEGG pathway analysis for immune response associated different identified proteins. c, The analyses of functional interaction network of identified proteins regulated genes by using the Search Tool for the Retrieval of Interacting Genes/Proteins (STRING) algorithm. d, Venn diagram of a primary transcript among identified proteins interacted with Tas of PFV, SFVora and SFV agm. 
a
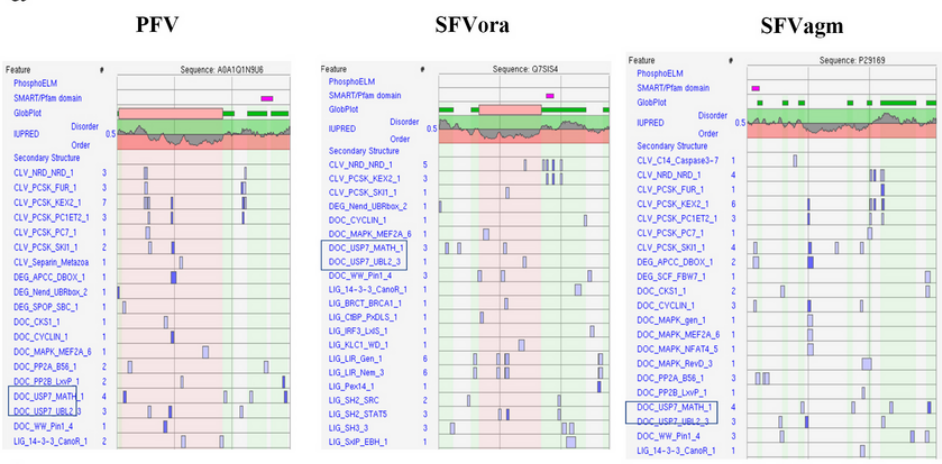

b
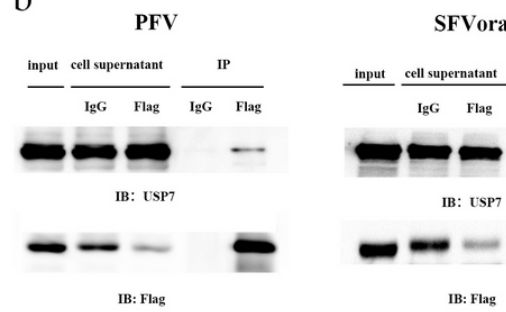

Vora

SFVagm

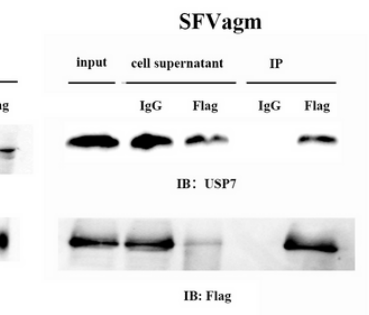

C

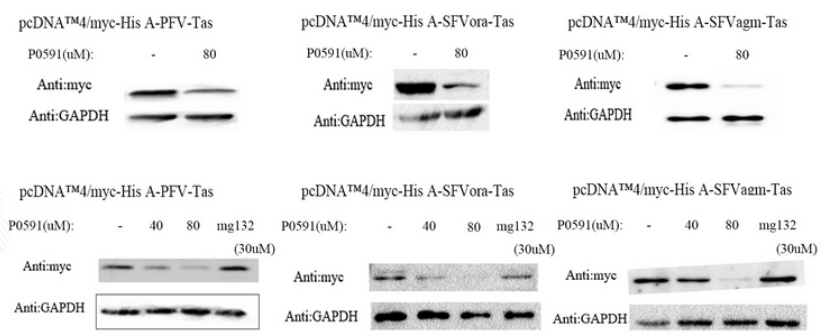

\section{Figure 6}

Interactions with USP7 illustrated the ubiquitin-dependent proteasome degradation of the Tas. a, EML (http://elm.eu.org) was used to discover functional linear motifs of Tas that potentially interacted Eukaryotic cytokines. b,Co-immunoprecipitation was performed to test the interaction between different Tas and USP7. Hela- SFVagm-3×Flag-Tas, Hela-SFVora-3×Flag-Tas, Hela- PFV-3×Flag-Tas cells were seeded into 10 -cm dish for $24 \mathrm{~h}$ and then added Dox into the cell medium at $1 \mathrm{ug} / \mathrm{ml}$ for the overexpression of Tas for $48 \mathrm{~h}$. Cell lysis was incubated with indicated antibodies Flag and normal igG (acted as control) overnight at $4^{\circ} \mathrm{C}$ and then rotated with Protein A-agarose, WB was performed to detected USP7 and Flag. c, The recombinant plasmid pcDNA ${ }^{\mathrm{TM}} 4 / \mathrm{myc}^{\mathrm{H}} \mathrm{His} \mathrm{A}$ was constructed to overexpress Tas of SFVagm, SFVora and PFV and were transfected into Hela cells. USP7 inhibitor P5091 and proteasomal inhibitor MG132 added into cell mediums at different concentrations. WB was performed to detected the expression of Tas.

\section{Supplementary Files}

This is a list of supplementary files associated with this preprint. Click to download.

- SupplementaryMaterial3.docx

- supplementarymaterials1.docx

- supplementarymaterials2.docx 